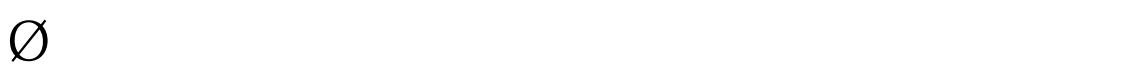

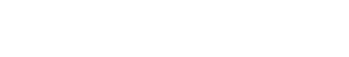 \\ GI由dŔC

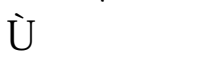

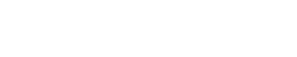

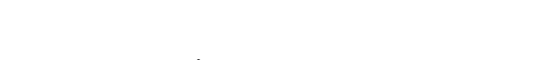

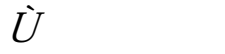

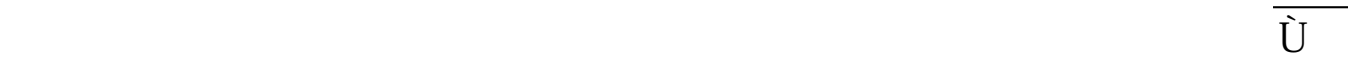
ŭ ŤBIE

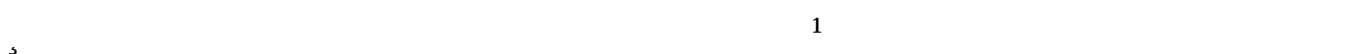

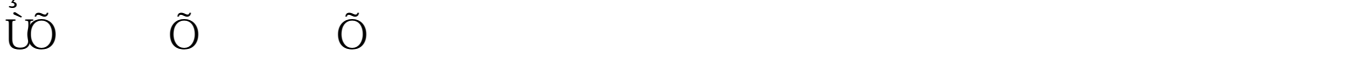

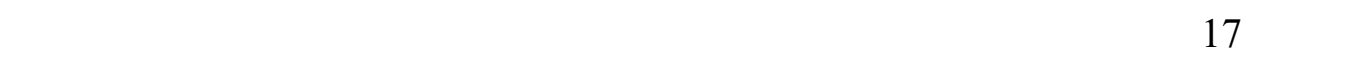

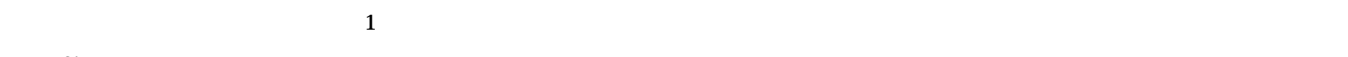

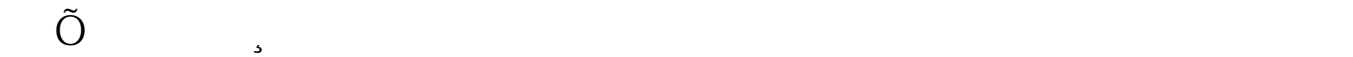

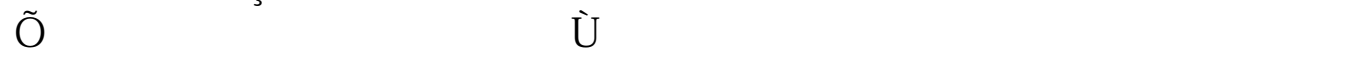

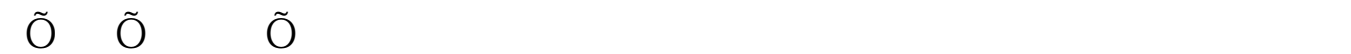
.RũÛ́

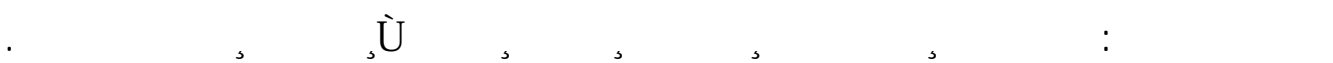

\section{Evaluation of Drinking Water Quality in Group of Schools at Mosul City}

\author{
Mazin nazzar Al-Sanjari \\ Environmental Research center \\ University of Mosul
}

\author{
Musa'b A. Al-Tamir \\ college of Engineering/Civil dept \\ University of Mosul
}

\section{ABSTRACT}

The monitoring of water quality produced for drinking is one of the most important study in order to evaluate the suitability of water for human consumption also to evaluate efficiencies of treatment water plants and water distribution system especially in the present situation of the country. This study focusing on the quality of drinking water used by student in some schools at Mosul city. Where 17 schools had been selected through the city. main chemical and physical analysis had been done on these samples in addition to the total bacteria count, the study revealed that the water quality of the studied schools was within the standards of drinking water. A negative result were appeared for total bacteria count in one sample, only seem to be an indication of a new case of pollution with bacteria, this might be due to the water supply network, fortunately the result were within the Iraqi standards for drinking water.

Key words: Water quality, Drinking water, Schools, Iraq, Mosul, Blockade, Water Supply. 


\begin{tabular}{llll} 
Al-Rafidain Engineering & Vol.14 & No.3 & 2006 \\
\hline
\end{tabular}

\section{rithe}

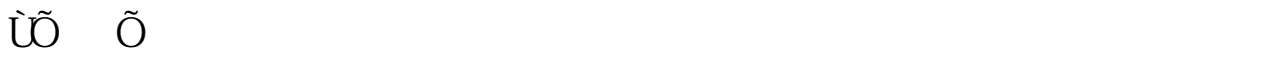

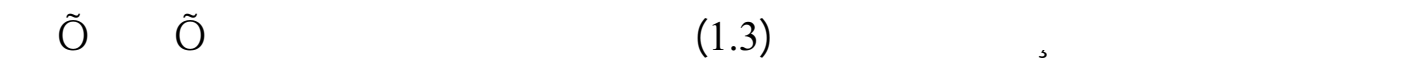

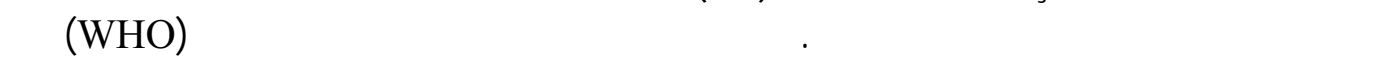

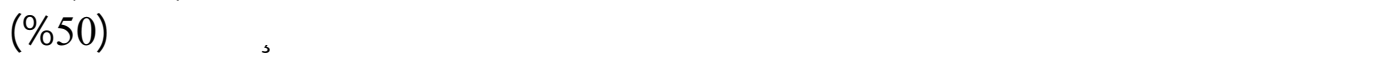

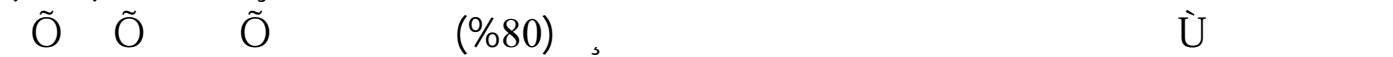

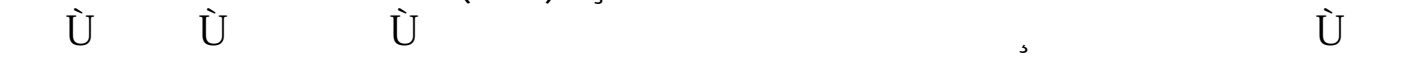

[2] [1].řǓ́fłtí

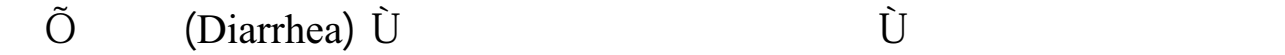

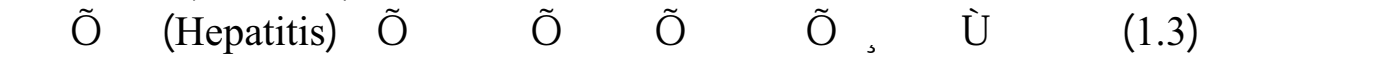

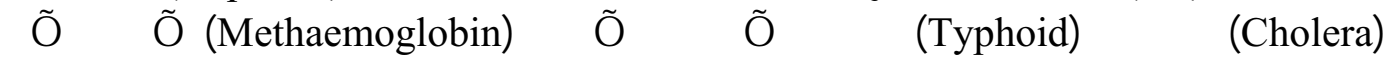

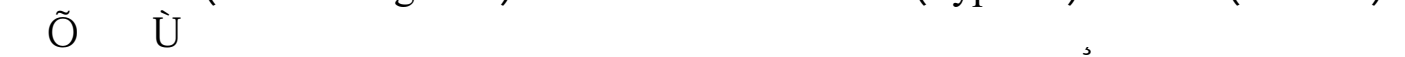

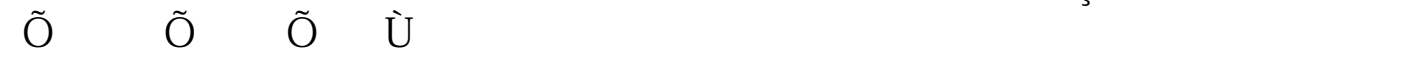

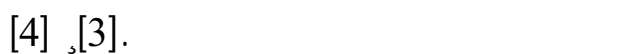

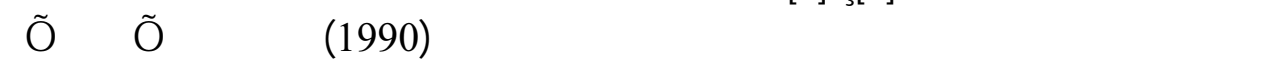

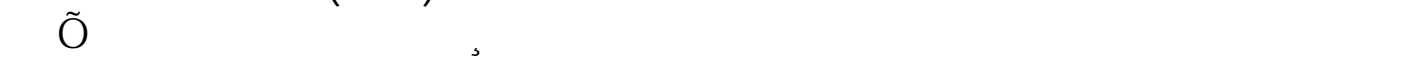

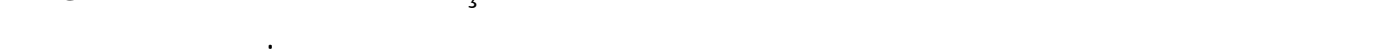

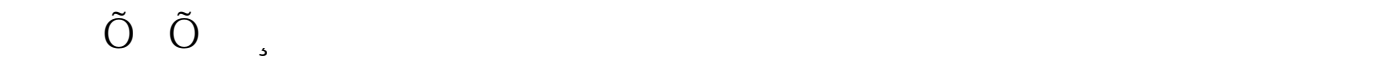

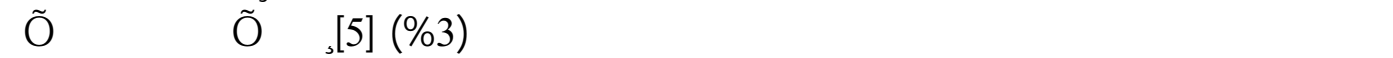

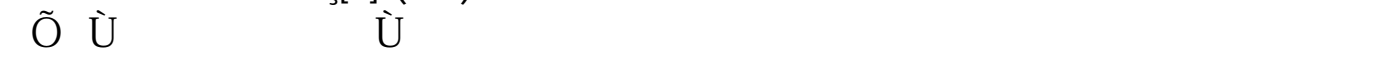

[7][6] .1988 đ̂y Gžzt

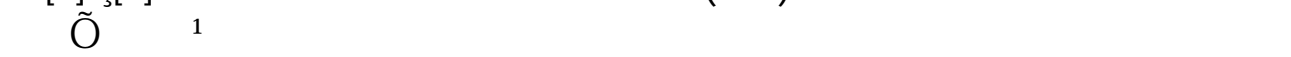
ũy

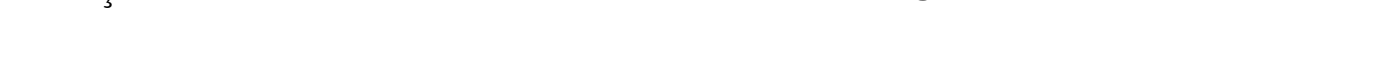

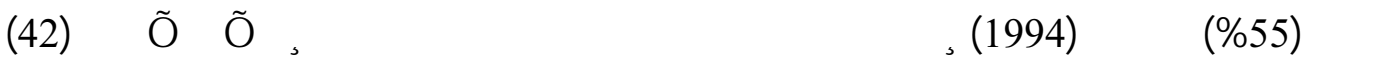

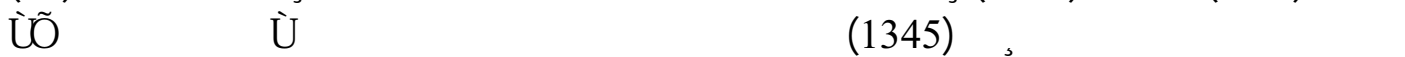

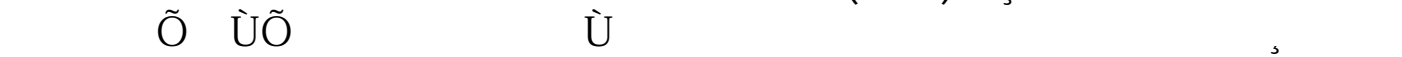

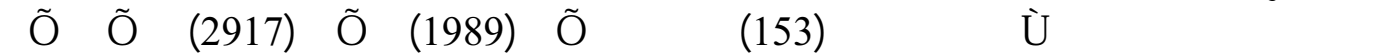
[6].C(1998)

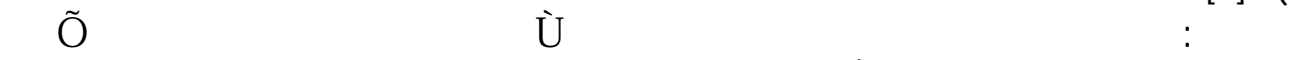

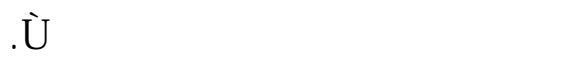

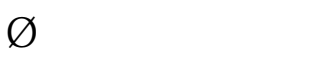

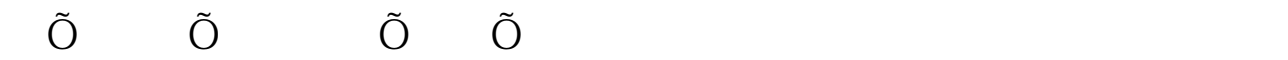

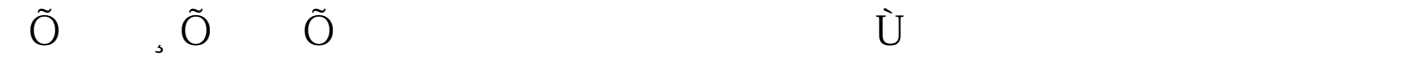

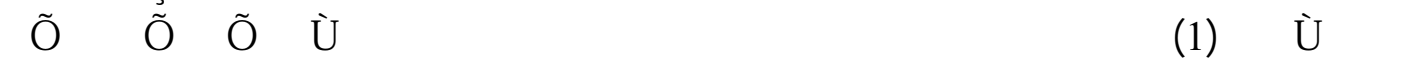

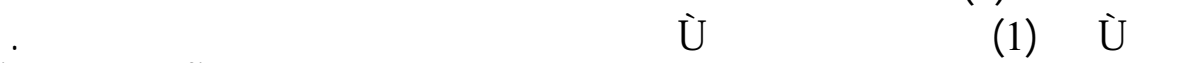

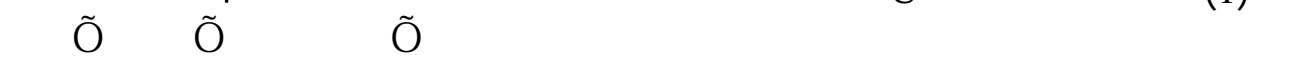

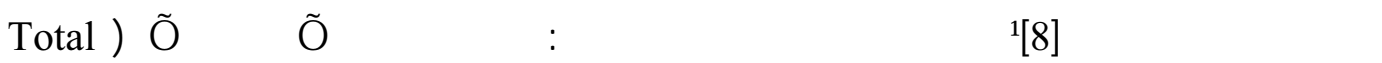

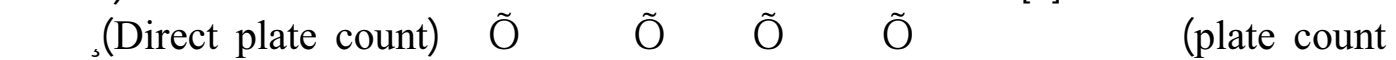

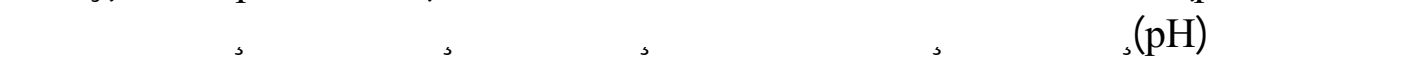




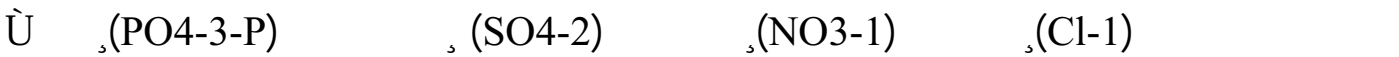

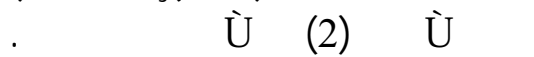

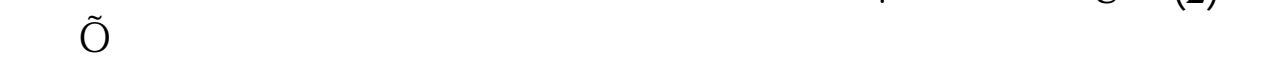

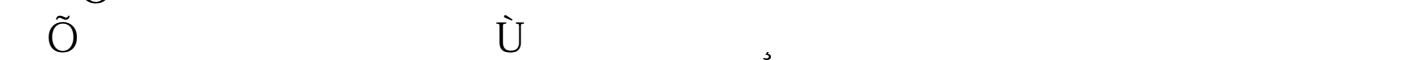

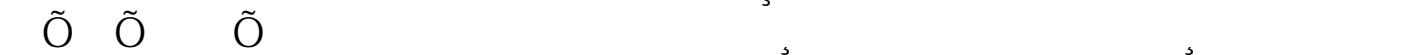

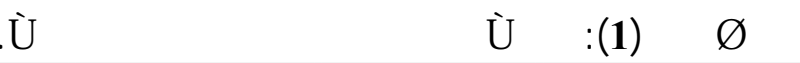

\begin{tabular}{|c|c|c|c|c|c|}
\hline 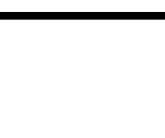 & 窇 & 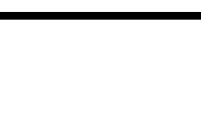 & rhïcose & rūetzraing & 葛 \\
\hline EREF ' 'níc & ũuัýR & 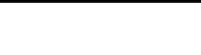 & 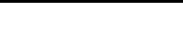 & Gỡddérữuté & 1 \\
\hline ươnct'U & ũŭÝR & 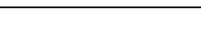 & 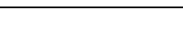 & ŚfựDu & 2 \\
\hline EÁRF 'níc & ũưÝ̛R & 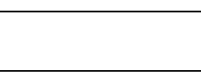 & 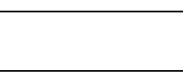 & 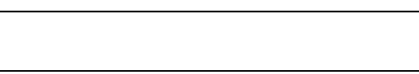 & 3 \\
\hline EÁEF 'níć & ũŭÝR & 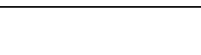 & Ŝिप̈tík & 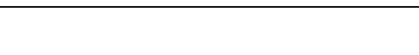 & 4 \\
\hline 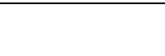 & ũư̛̆́́ & 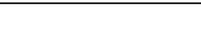 & 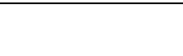 & 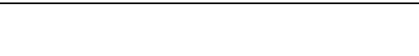 & 5 \\
\hline EÁRF 'ní́ & ũưÝ̛R & 䒮宜 & ũç̋ Ğ Ģ & 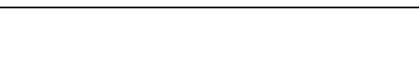 & 6 \\
\hline EÁEF 'níc & ũưÝ̛R & 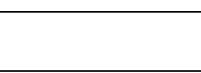 & 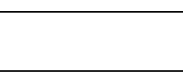 & 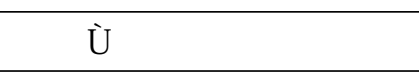 & 7 \\
\hline EÁ̈F 'níc' & ũǒÝR & 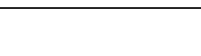 & 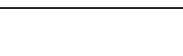 & 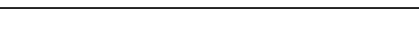 & 8 \\
\hline 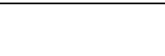 & ũư̆́R & 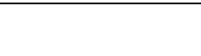 & 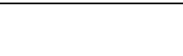 & 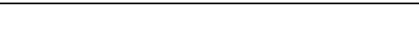 & 9 \\
\hline EREF 'ńć & ũưÝ̛ & 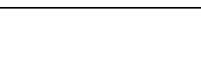 & 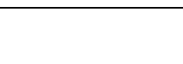 & 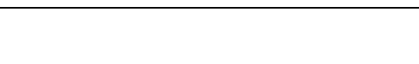 & 10 \\
\hline EÁRF 'níć & ũưđ̛́ & 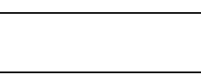 & 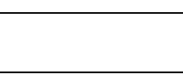 & 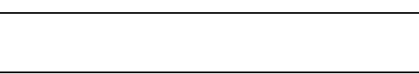 & 11 \\
\hline EÁEF 'ní́c & ũưÝ̛ & 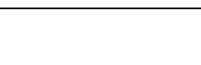 & ũagūíḱç & 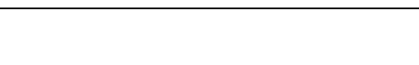 & 12 \\
\hline ũợịct t'บ & ECYŔ & ţ̦ ZdR'́nídŔ & Las 17 & EUR日 & 13 \\
\hline ũợịct t'บ & ECYŔ & ț ZdR'́nídŔ & Gŷĩũú & 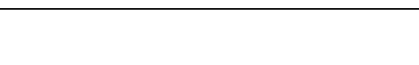 & 14 \\
\hline ũonct t'U & ĐCYḰ & 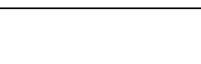 & 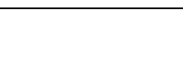 & 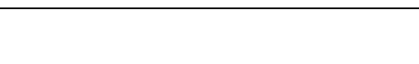 & 15 \\
\hline EREF 'ńć & ECYḰ & 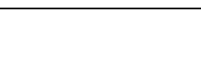 & 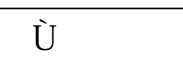 & 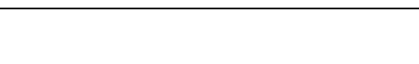 & 16 \\
\hline ERिA 'níć & ECYḰ & 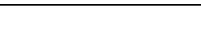 & 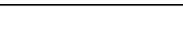 & Expoyit rystín & 17 \\
\hline
\end{tabular}




\begin{tabular}{llll} 
Al-Rafidain Engineering & Vol.14 & No.3 & 2006 \\
\hline
\end{tabular}

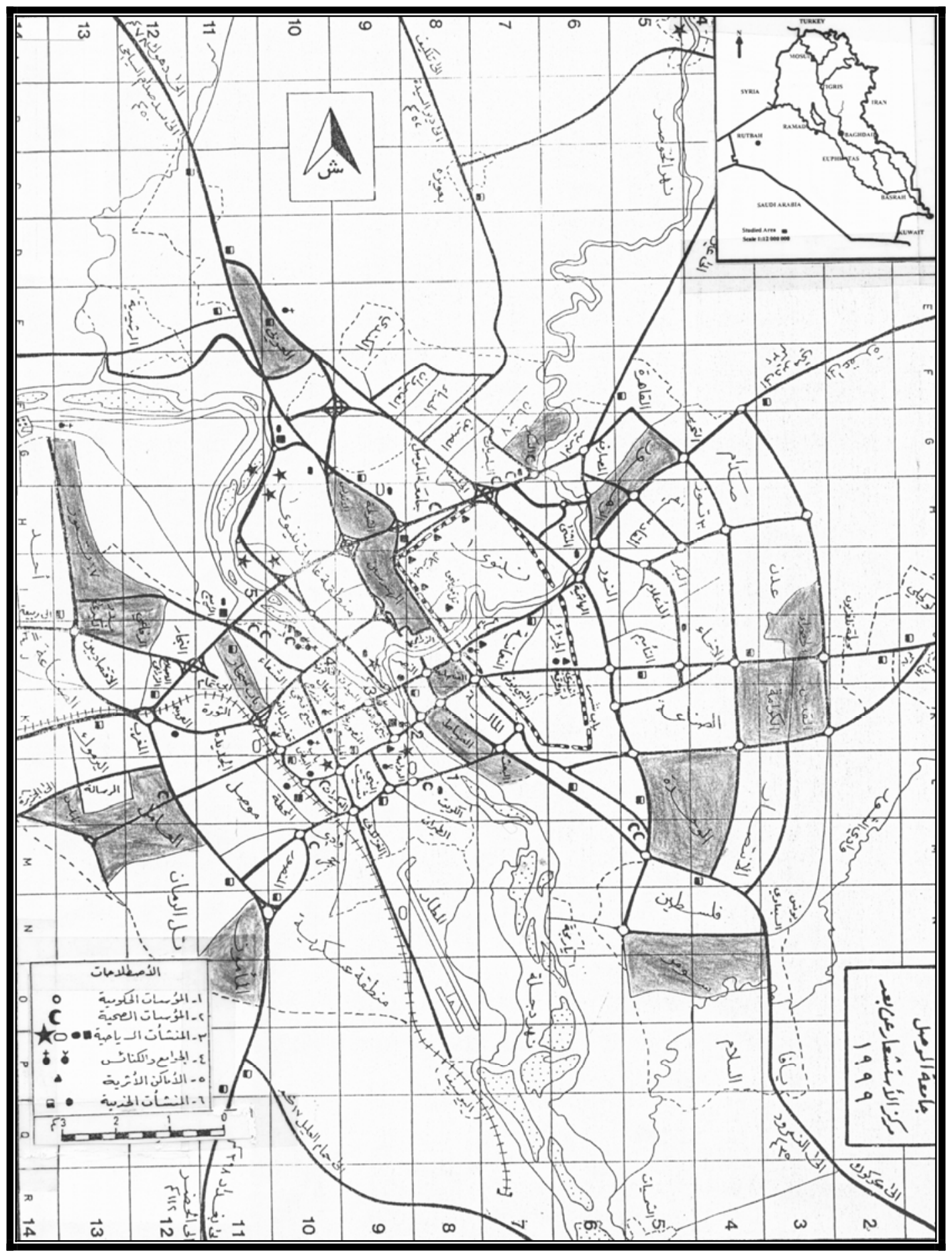

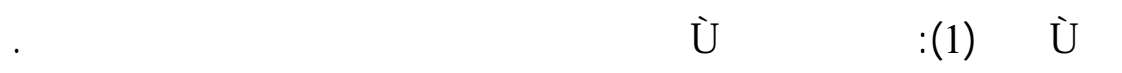




\section{rǘrểrE}

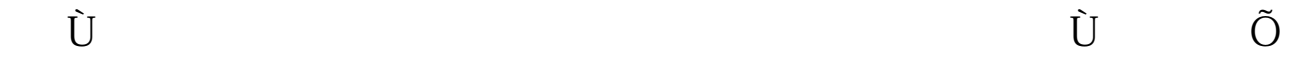

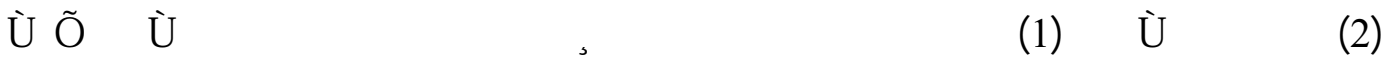

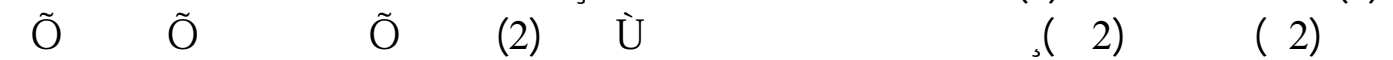

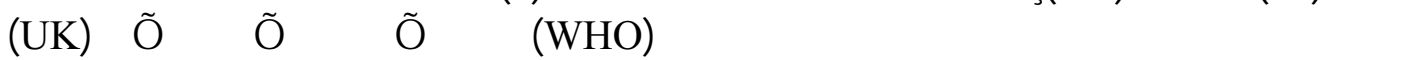

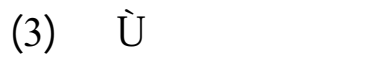

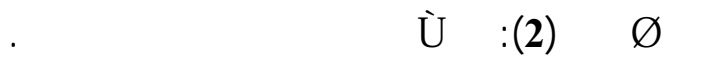

\begin{tabular}{|c|c|c|c|c|c|c|c|c|c|c|}
\hline 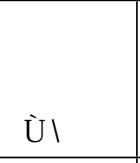 & $\begin{array}{l}\mathrm{PO}_{4}^{-3} \\
\mathrm{mg} / 1\end{array}$ & $\begin{array}{c}\mathrm{SO}_{4}^{-2} \\
\mathrm{mg} / 1\end{array}$ & $\begin{array}{c}\mathrm{NO}_{3}^{-1} \\
\mathrm{mg} / 1\end{array}$ & $\begin{array}{l}\mathrm{Cl}^{-1} \\
\mathrm{mg} / \mathrm{l}\end{array}$ & $\begin{array}{l}\text { řłŷn } \\
\mathrm{mg} / \mathrm{l} \text { as } \\
\mathrm{CaCO}_{3}\end{array}$ & $\begin{array}{l}\text { F̌ı̛̆̈ß́ } \\
\mathrm{mg} / 1 \text { as } \\
\mathrm{CaCO}_{3}\end{array}$ & 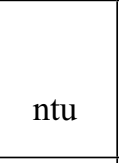 & $\begin{array}{c}\text { EC. } \\
\mu \mathrm{S} / \mathrm{cm} \\
@ \\
25^{\circ} \mathrm{C} \\
\end{array}$ & $\mathrm{pH}$ & $\Sigma$ \\
\hline 0 & 0.027 & 56 & 0.35 & 11 & 120 & 225 & 1.4 & 421 & 7.93 & 1 \\
\hline 0 & 0.03 & 56 & 0.31 & 10 & 140 & 230 & 2.2 & 394 & 7.9 & 2 \\
\hline 0 & 0.04 & 61 & 0.3 & 11 & 150 & 235 & 2.5 & 422 & 7.87 & 3 \\
\hline 36 & 0.012 & 55 & 0.33 & 10 & 150 & 230 & 1.9 & 423 & 7.93 & 4 \\
\hline 0 & 0.017 & 58 & 0.36 & 12 & 120 & 220 & 0.6 & 418 & 7.89 & 5 \\
\hline 0 & 0.06 & 56 & 0.33 & 11 & 120 & 220 & 0.8 & 416 & 7.91 & 6 \\
\hline 0 & 0.03 & 58 & 0.33 & 10 & 125 & 220 & 1.2 & 390 & 7.92 & 7 \\
\hline 0 & 0.07 & 56 & 0.4 & 11 & 120 & 235 & 0.6 & 419 & 7.89 & 8 \\
\hline 0 & 0.007 & 56 & 0.36 & 9 & 110 & 225 & 0.7 & 410 & 7.91 & 9 \\
\hline 0 & 0.012 & 65 & 0.3 & 10 & 125 & 235 & 1 & 419 & 7.65 & 10 \\
\hline 0 & 0.008 & 57 & 0.38 & 11 & 115 & 230 & 1.2 & 418 & 7.93 & 11 \\
\hline 0 & 0.042 & 63 & 0.34 & 12 & 115 & 230 & 1.4 & 441 & 7.94 & 12 \\
\hline 0 & 0.025 & 72 & 0.29 & 11 & 115 & 235 & 5 & 349 & 7.66 & 13 \\
\hline 0 & 0.01 & 71 & 0.33 & 10 & 115 & 270 & 3.8 & 438 & 7.65 & 14 \\
\hline 0 & 0.012 & 72 & 0.33 & 11 & 130 & 265 & 2.1 & 433 & 7.65 & 15 \\
\hline 0 & 0.01 & 56 & 0.29 & 11 & 110 & 215 & 0.6 & 407 & 7.95 & 16 \\
\hline 0 & 0.006 & 63 & 0.32 & 10 & 115 & 225 & 2 & 416 & 7.84 & 17 \\
\hline
\end{tabular}

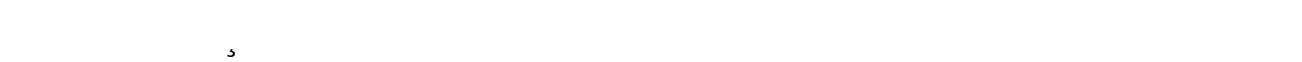

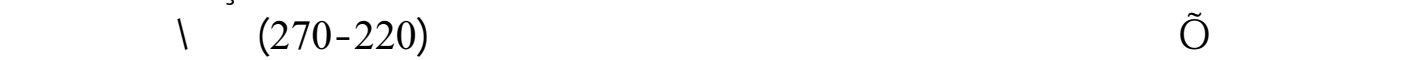

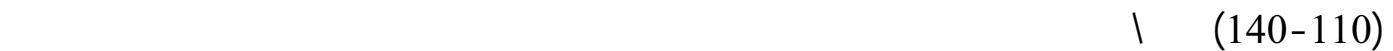

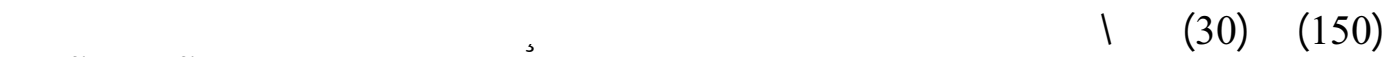

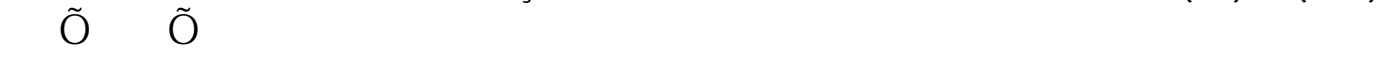

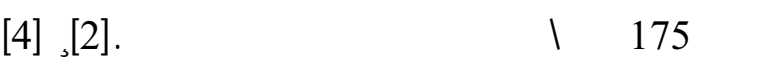

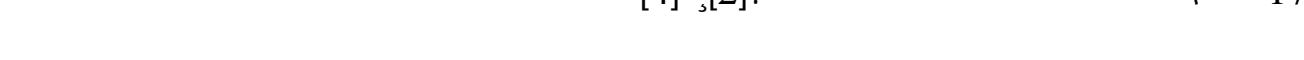

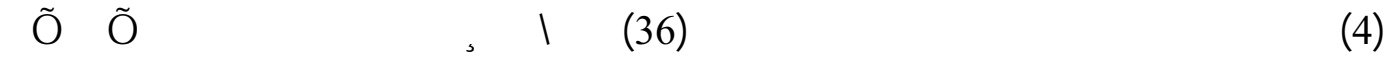

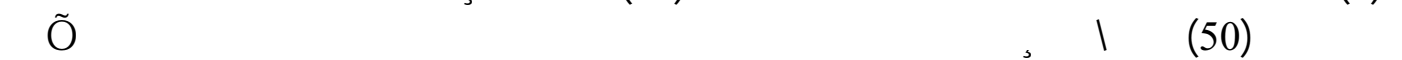

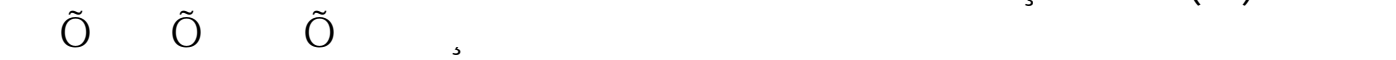

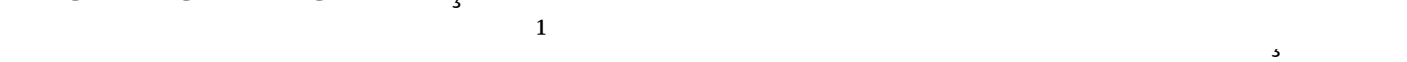




$\begin{array}{llll}\text { Al-Rafidain Engineering } & \text { Vol.14 } & \text { No.3 } & 2006\end{array}$

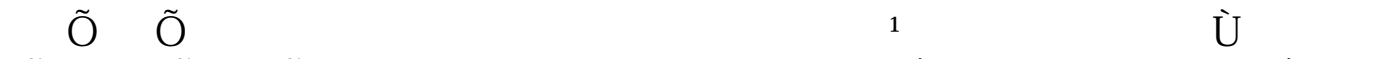

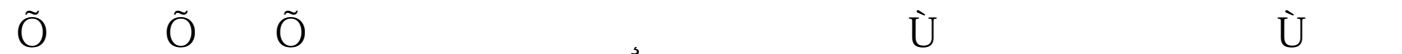

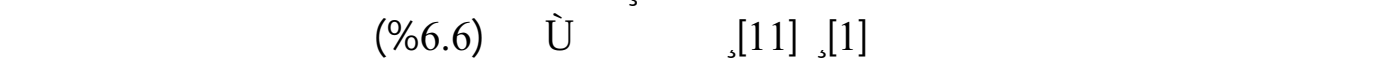

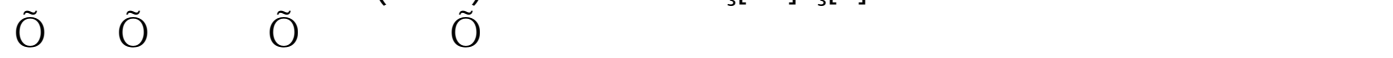
.ryuśstís

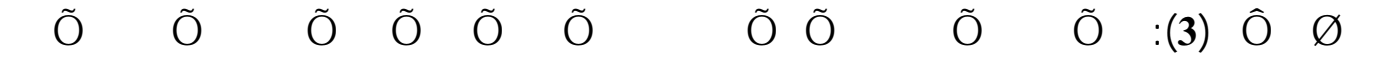

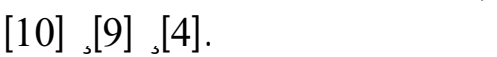

\begin{tabular}{|c|c|c|c|}
\hline 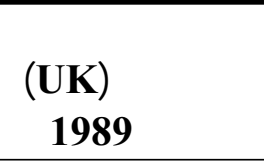 & 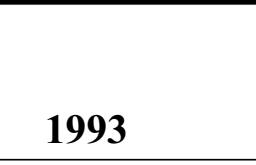 & 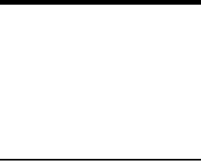 & !̣UீăăE \\
\hline $9.5-5.5$ & $<8$ & $8.5-6.5$ & 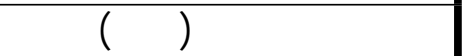 \\
\hline 1635 & - & - & 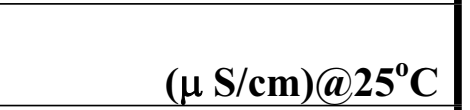 \\
\hline 4 & 5 & 5 & (ntu) Regin \\
\hline 400 & 250 & 200 & $(\mathrm{mg} / \mathrm{l})\left(\mathrm{Cl}^{-1}\right) \hat{\mathrm{R}} \mathrm{fg}$ \\
\hline 50 & 50 & 20 & $(\mathrm{mg} / \mathrm{l})\left(\mathrm{NO}_{3}{ }^{-1}\right) \tilde{\mathrm{R}} \mathrm{C}$ \\
\hline 250 & 250 & 200 & 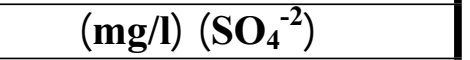 \\
\hline 2.2 & - & - & 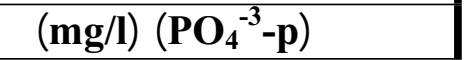 \\
\hline 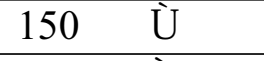 & - & 500 & $\left(\mathrm{mg} / \mathrm{l}\right.$ as $\left.\mathrm{CaCO}_{3}\right)$ Rế, \\
\hline 30 Êे ¿ E⿹ & - & 170 & $\left(\mathrm{mg} / \mathrm{l}\right.$ as $\left.\mathrm{CaCO}_{3}\right)$ rftîntra \\
\hline 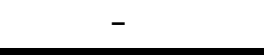 & - & 50 & 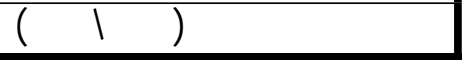 \\
\hline
\end{tabular}

\section{Řş̌́tưke}

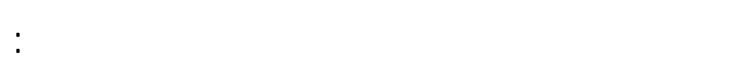

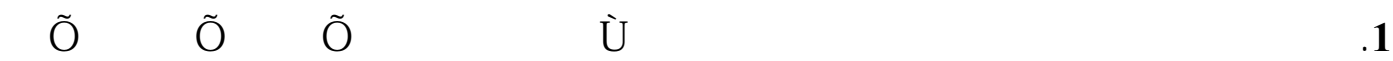
.Gร์วอัง์

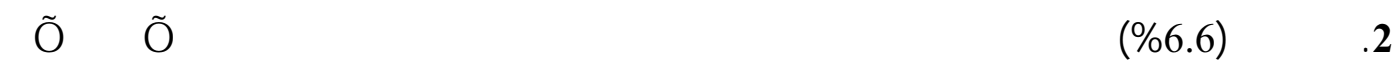

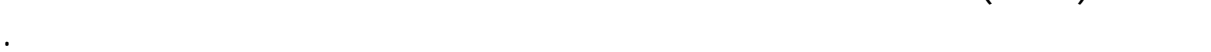

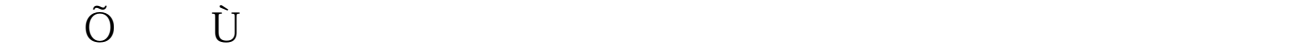

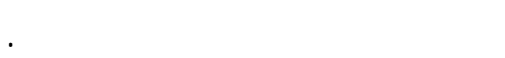

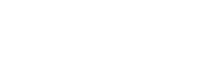

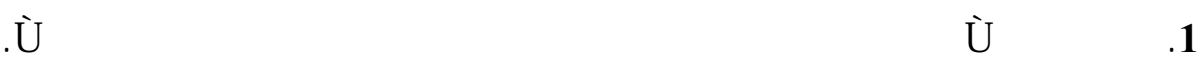

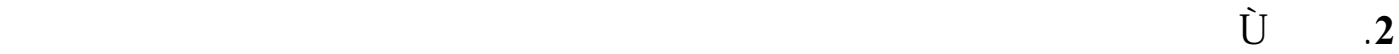

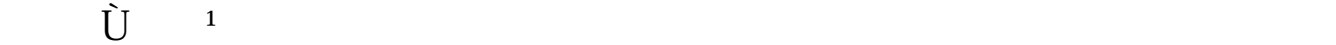

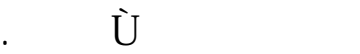

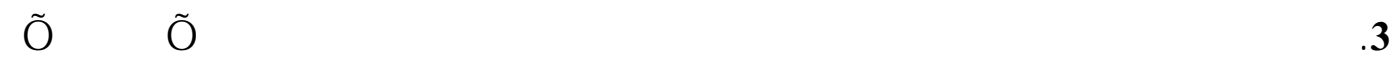



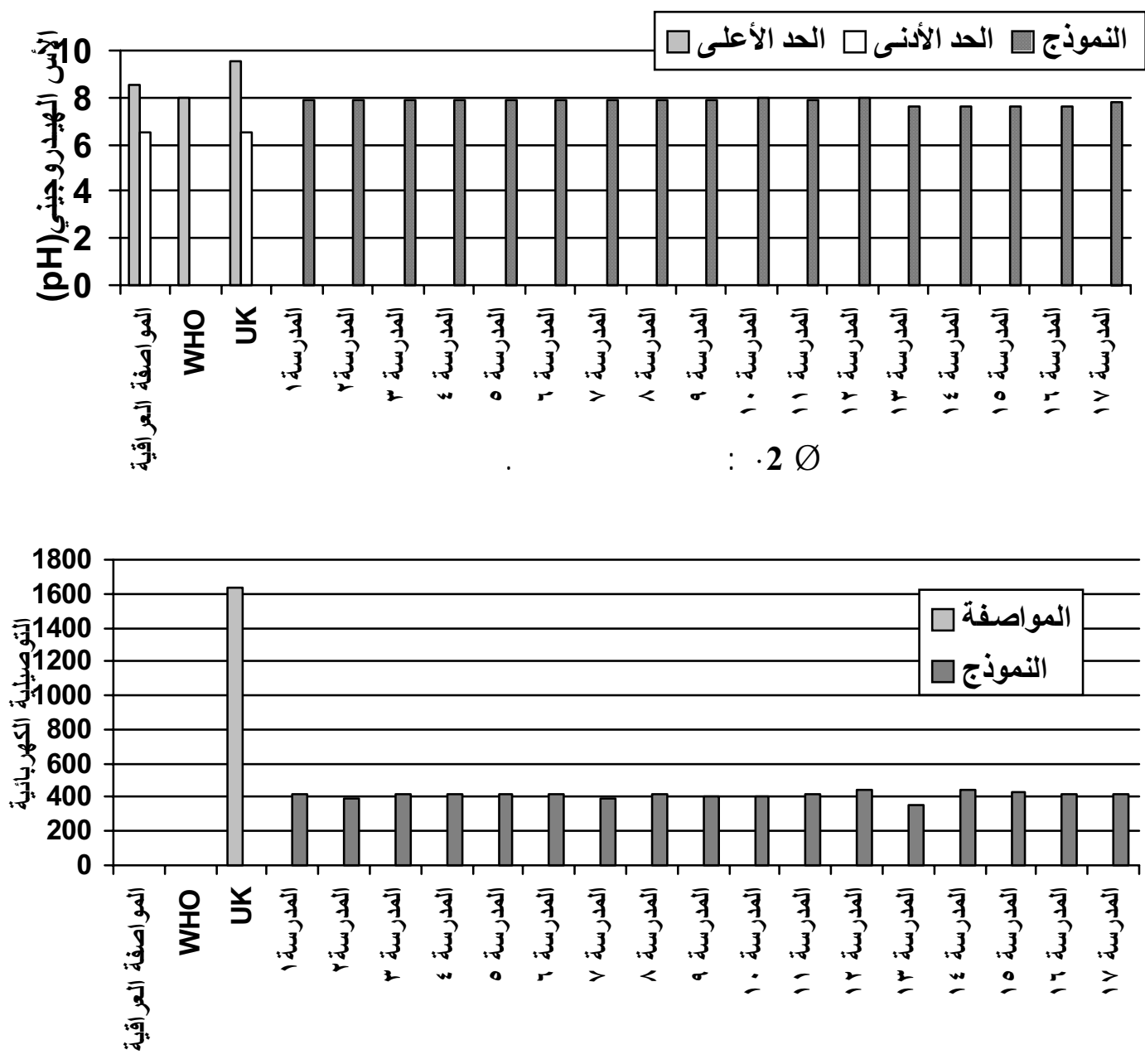

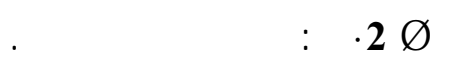

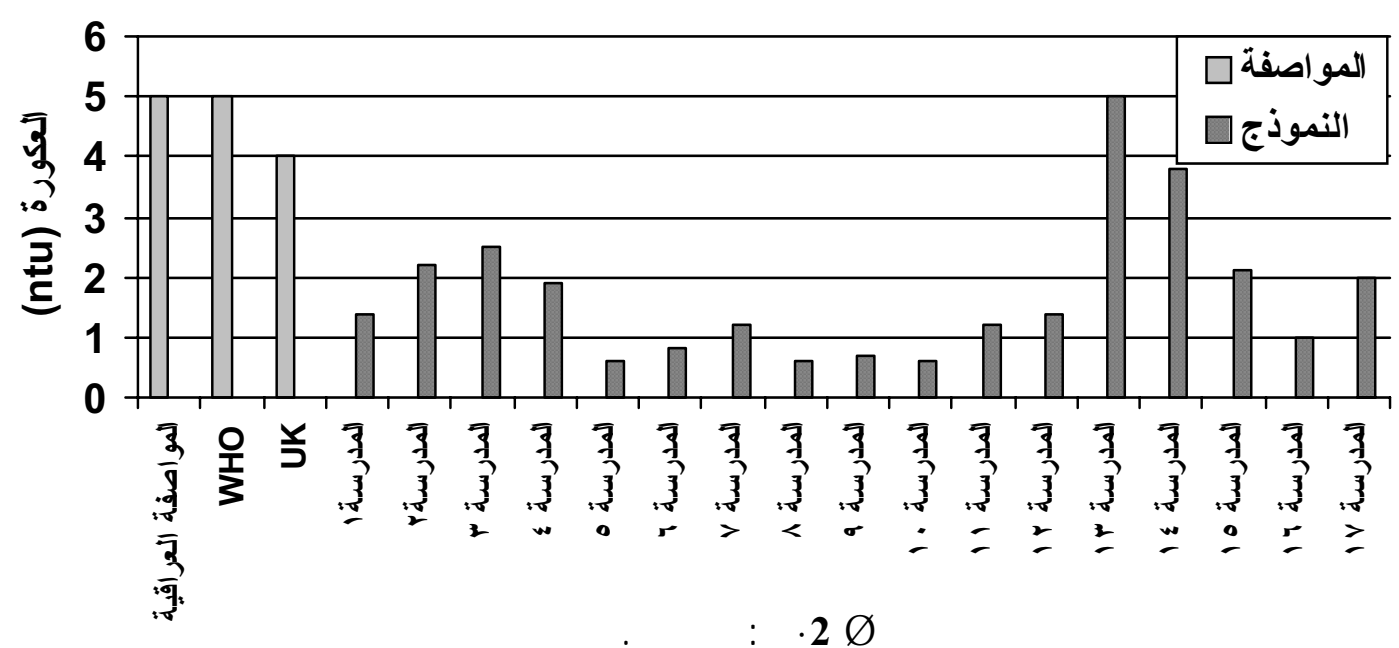




\begin{tabular}{llll} 
Al-Rafidain Engineering & Vol.14 & No.3 & 2006 \\
\hline
\end{tabular}
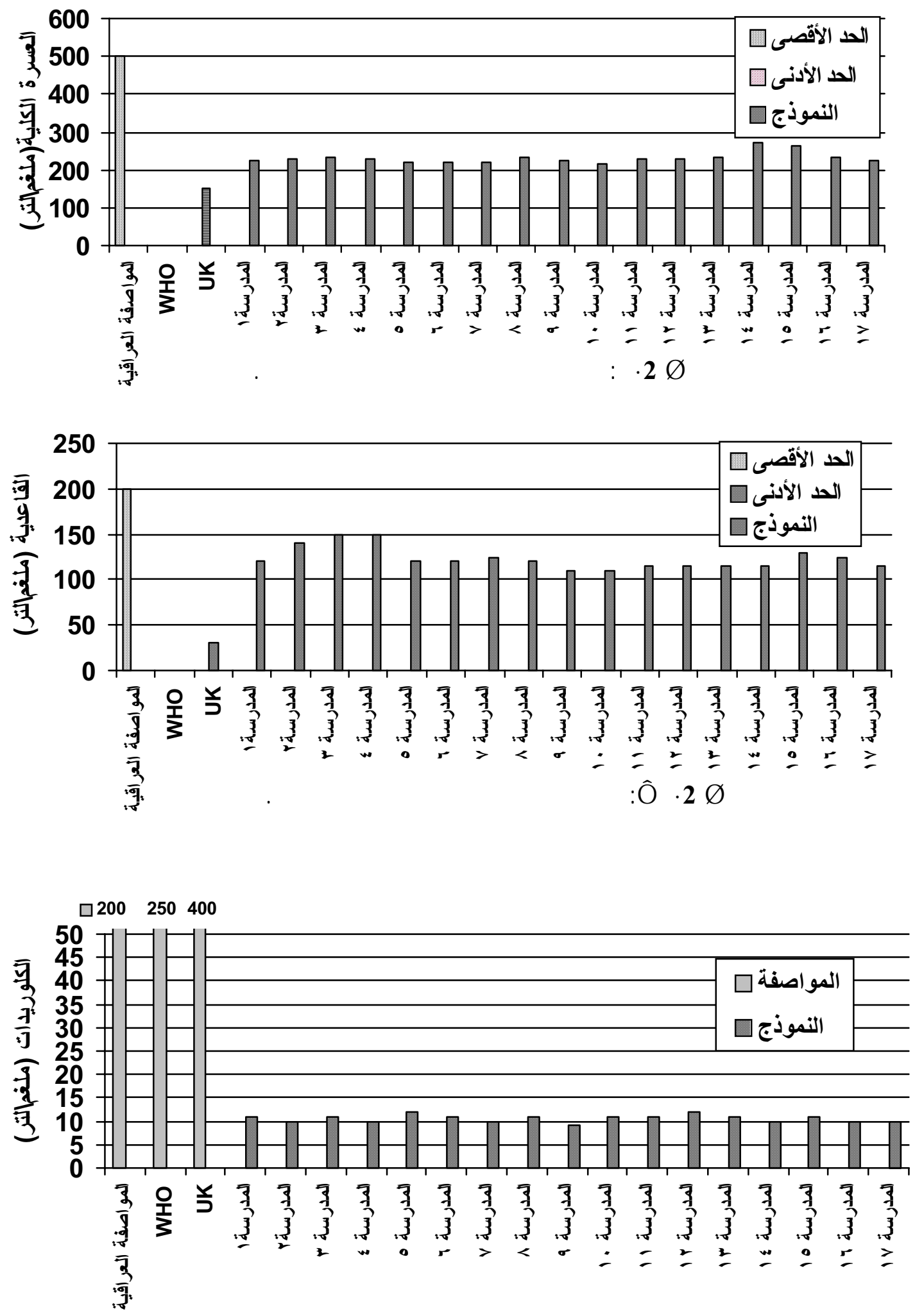

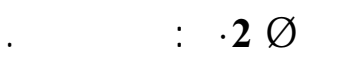



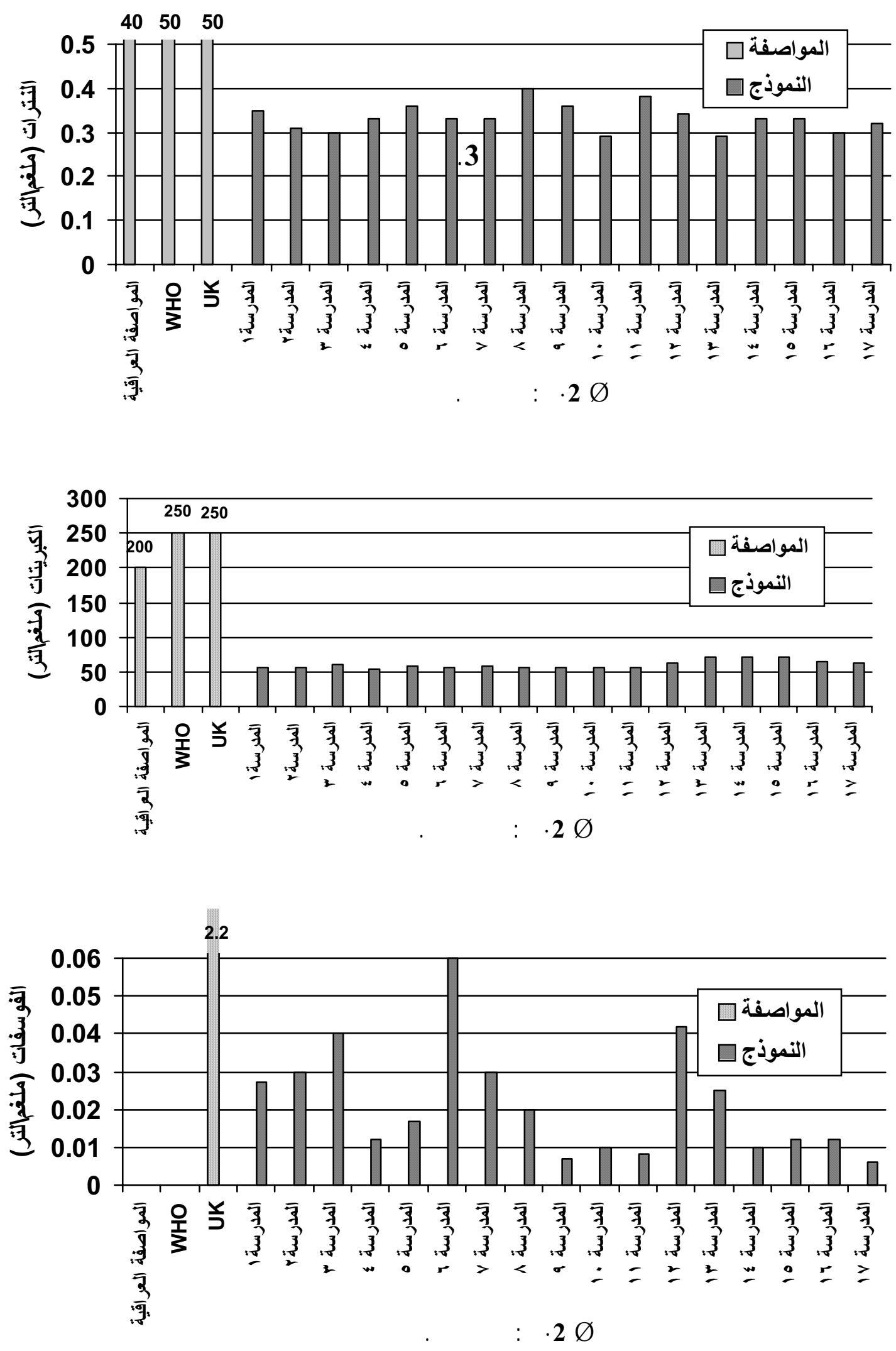


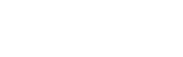

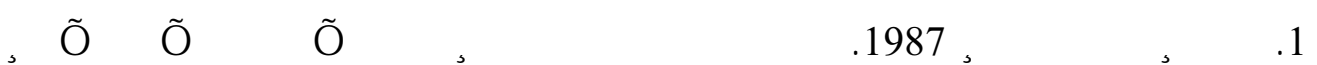

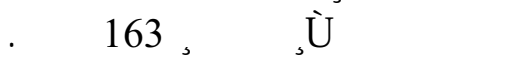

2. Tebbutt, T. H. Y., 1998. Principles of Water Quality Control, $5^{\text {th }}$ ed., Butter worth- heinemann, Oxford, USA, 280p.

3. McGhee, T. J., 1991. Water Supply and Sewerage, $6^{\text {th }}$ ed., McGraw-Hill, Inc., New york, USA, 602p.

4. Twort, A. C., Ratnayaka, S. D. and Brandt, M. J., 2001. Water Supply, $5^{\text {th }}$ ed., Butter worth- heinemann, Oxford, USA, 676p.

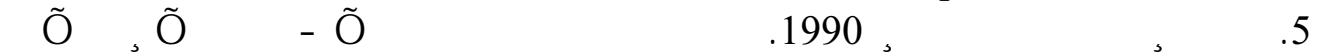

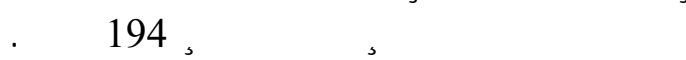

6. htt//www.aljazeera.net/in-depth/iraq blockade/2003/3/3-18-1.htm

7. http//www.albaycen.coae/albayan/alarabea/2003/isswe/orbit/1.htm

8. APHA, AWWA, WPCF, 1985. Standard Method for the Examination of Water and Wastewater, $16^{\text {th }}$ ed., NewYork, 1268p.

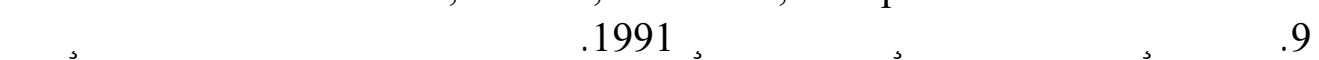

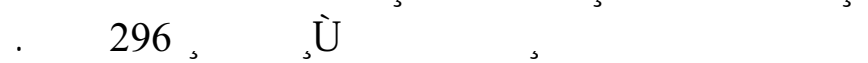

10. WHO, 1997. Giudlines for drinking water quality Health Criteria and Other Supporting Information, Vol.2, $2^{\text {nd }}$ ed. Geneva, (CEHA), Amman, Jordan, 973p.

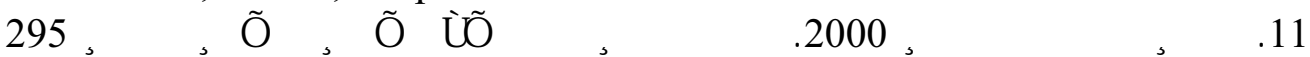
. řţü 